\title{
CO-driven activity constrains the origin of comets
}

\author{
M. Fulle ${ }^{1}$, J. Blum ${ }^{2}$, and A. Rotundi ${ }^{3}$ \\ 1 INAF - Osservatorio Astronomico, Via Tiepolo 11, 34143 Trieste, Italy \\ e-mail: marco.fulle@inaf.it \\ 2 Inst. für Geophysik und extraterrestrische Physik, Techn. Univ. Braunschweig, Mendelssohnstr. 3, 38106 Braunschweig, Germany \\ 3 Universitá degli Studi di Napoli Parthenope, Dip. di Scienze e Tecnologie, CDN IC4, 80143 Naples, Italy
}

Received 24 February 2020 / Accepted 11 March 2020

\begin{abstract}
Context. An open question in the study of comets is the so-called cohesion bottleneck, that is, how dust particles detach from the nucleus.

Aims. We test whether the $\mathrm{CO}$ pressure buildup inside the pebbles of which cometary nuclei consist can overcome this cohesion bottleneck.

Methods. A recently developed pebble-diffusion model was applied here to comet C/2017K2 PANSTARRS, assuming a CO-driven activity.

Results. (i) The CO-gas pressure inside the pebbles erodes the nucleus into the observed dust, which is composed of refractories, $\mathrm{H}_{2} \mathrm{O}$ ice and $\mathrm{CO}_{2}$ ice. (ii) The CO-driven activity onset occurs up to heliocentric distances of 85 au, depending on the spin orientation of the comet nucleus. (iii) The activity onset observed at $\approx 26$ au suggests a low obliquity of the nucleus spin axis with activity in a polar summer. (iv) At 14 au, the smallest size of the ejected dust is $\approx 0.1 \mathrm{~mm}$, consistent with observations. (v) The observed dust-loss rate of $\approx 200 \mathrm{~kg} \mathrm{~s}^{-1}$ implies a fallout $\geq 30 \%$, a nucleus surface active area $\geq 10 \mathrm{~km}^{2}$, a CO-gas loss rate $\geq 10 \mathrm{~kg} \mathrm{~s}^{-1}$, and a dust-to-gas ratio $\leq 20$. (vi) The CO-driven activity never stops if the average refractory-to-all-ices mass ratio in the nucleus is $\leq 4.5$ for a nucleus all-ices-to-CO mass ratio $\approx 4$, as observed in comets Hale-Bopp and Hyakutake. These results make comet C/2017K2 similar to the Rosetta target comet 67P/Churyumov-Gerasimenko. (vii) The erosion lifetime of cometary planetesimals is a factor $10^{3}$ shorter than the timescale of catastrophic collisions. This means that the comets we observe today cannot be products of catastrophic collisions.
\end{abstract}

Key words. comets: general - comets: individual: C/2017K2 PANSTARRS - Oort Cloud - protoplanetary disks

\section{Introduction}

While the activity of comets inside the Jupiter orbit is mainly driven by water-ice sublimation, beyond Saturn, this activity is driven by the so-called supervolatiles, of which $\mathrm{CO}$ is the most abundant (Bockelee-Morvan et al. 2004). A detailed characterization of the CO-driven activity of comets is needed also in view of the ESA mission Comet Interceptor (Snodgrass \& Jones 2019), which will target an Oort-cloud (or even interstellar) comet discovered as far as possible from the Sun. These models will support the choice of the probe trajectory to best fly-by its target.

Of the comets that are active beyond Saturn the incoming comet C/2017K2 PANSTARRS was discovered at 16 au from the Sun, with prediscovery images at $24 \mathrm{au}$, when it was already active, and a period of $\approx 3 \mathrm{Myr}$ consistent with inner temperatures of a few K, typical of Oort comets before the first Sun approach (Jewitt et al. 2019). Models of the collected data suggest an activity onset at about 26 au from the Sun and a dust coma made of particles larger than $0.1 \mathrm{~mm}$, released at a steady rate of $\approx 200 \mathrm{~kg} \mathrm{~s}^{-1}$ (Jewitt et al. 2019). Beyond Saturn, the gas temperature and density at the nucleus surface are too low to detach the observed dust from the nucleus; tensile strengths $>0.1 \mathrm{~Pa}$ bond each particle to the nucleus (Skorov \& Blum 2012). Therefore, exotic processes (thermal stress or electrostatic charging) have been invoked to explain the dust ejection (Jewitt et al. 2019).

The dust cohesion bottleneck is a well-known paradox in cometary physics and has been explained only recently for a water-driven cometary activity (Fulle et al. 2020). Here, we apply this model to CO-driven activity, showing that the CO-gas pressure inside the pebbles of which cometary nuclei consist (Blum et al. 2017) explains the ejection of the observed dust and fits all the observed data. Here we make the following assumptions for CO: (i) its fraction trapped in other ices and clathrates is negligible, and (ii) it is present as pure ice, thus sublimating beyond Saturn. We find that the Oort comet C/2017K2 is similar to all Jupiter-family comets: it has high values of the refractory-to-all-ices mass ratio and of dust fallout (Fulle et al. 2019). Our results also constrain the evolution of cometary planetesimals.

\section{Activity model}

The first thermal model consistent with dust ejection from cometary nuclei (Fulle et al. 2020) is applied here to the specific case of a comet with CO-driven activity at heliocentric distances that are too large to allow any significant sublimation of $\mathrm{H}_{2} \mathrm{O}$ and $\mathrm{CO}_{2}$ ices. The model is consistent with a nucleus consisting of centimeter-sized pebbles (Blum et al. 2017) where ice sublimation occurs only inside the pebbles (Fulle et al. 2020). Pebbles are inhomogeneous clusters of dust particles, which are porous aggregates of ice and dust grains (Levasseur-Regourd et al. 2018; Güttler et al. 2019) and have a differential size distribution with a power index of $-3 \pm 1$ (Blum et al. 2017). In this specific case, dust is composed not only of refractories, as usual, but also of $\mathrm{H}_{2} \mathrm{O}$ and $\mathrm{CO}_{2}$ ices. All the governing equations we apply here with parameters valid for $\mathrm{CO}$ have been obtained 
Table 1. Symbols.

\begin{tabular}{|c|c|c|}
\hline Symbol & Definition & units \\
\hline$A$ & Bond albedo of the nucleus & \\
\hline$\epsilon$ & Emissivity of the nucleus & \\
\hline$\theta$ & Sun zenithal angle on the nucleus surface & \\
\hline$\delta$ & Refractory-to-all-ices mass ratio & \\
\hline$\gamma$ & All-ices-to-CO mass ratio & \\
\hline$r_{\mathrm{h}}$ & Nucleus heliocentric distance & $\mathrm{au}$ \\
\hline$r$ & Average radius of the dust grains & $\mathrm{m}$ \\
\hline$R$ & Average radius of the pebbles & $\mathrm{m}$ \\
\hline$s$ & Depth from the nucleus surface & $\mathrm{m}$ \\
\hline$s_{\mathrm{m}}$ & Diameter of the smallest ejected particles & $\mathrm{m}$ \\
\hline$s_{\mathrm{M}}$ & Diameter of the largest ejected particles & $\mathrm{m}$ \\
\hline$D$ & CO-depletion rate of the nucleus & $\mathrm{m} \mathrm{s}^{-1}$ \\
\hline$E$ & Erosion rate of the nucleus & $\mathrm{m} \mathrm{s}^{-1}$ \\
\hline$P$ & Pressure of CO gas inside the pebbles & $\mathrm{Pa}$ \\
\hline$S$ & Tensile strength bonding the dust grains & $\mathrm{Pa}$ \\
\hline$P_{0}$ & $\mathrm{CO}$-ice sublimation pressure & $\mathrm{Pa}$ \\
\hline$T_{0}$ & CO-ice sublimation temperature & K \\
\hline$T_{\mathrm{s}}$ & Temperature of the nucleus surface & $\mathrm{K}$ \\
\hline$\nabla T$ & Nucleus average temperature gradient & $\mathrm{Km}^{-1}$ \\
\hline$k$ & Boltzmann constant & $\mathrm{J} \mathrm{K}^{-1}$ \\
\hline$\Lambda$ & Sublimation latent heat of $\mathrm{CO}$ ice & $\mathrm{J} \mathrm{kg}^{-1}$ \\
\hline$c_{\mathrm{p}}$ & Heat capacity of the pebbles & $\mathrm{J} \mathrm{kg}^{-1} \mathrm{~K}^{-1}$ \\
\hline$\sigma$ & Stefan-Boltzmann constant & $\mathrm{W} \mathrm{m}^{-2} \mathrm{~K}^{-4}$ \\
\hline$I_{\odot}$ & Solar flux at $1 \mathrm{au}$ & $\mathrm{W} \mathrm{m}^{-2}$ \\
\hline$\lambda_{\mathrm{s}}$ & Heat conductivity inside the nucleus & $\mathrm{W} \mathrm{m} \mathrm{m}^{-1} \mathrm{~K}^{-1}$ \\
\hline$m$ & Mass of a CO molecule & $\mathrm{kg}$ \\
\hline$Q$ & Average flux of $\mathrm{CO}$ gas from the nucleus & $\mathrm{kg} \mathrm{m}^{-2} \mathrm{~s}^{-1}$ \\
\hline$Q_{\mathrm{d}}$ & Observed dust-loss rate from the nucleus & $\mathrm{kg} \mathrm{s}^{-1}$ \\
\hline$\rho_{\mathrm{d}}$ & Average bulk density of the dust particles & $\mathrm{kg} \mathrm{m}^{-3}$ \\
\hline$\rho_{\mathrm{n}}$ & Average bulk density of the nucleus & $\mathrm{kg} \mathrm{m}^{-3}$ \\
\hline
\end{tabular}

for a water-driven activity (Fulle et al. 2020). CO gas diffuses inside the pebbles, with a pressure inside the pebbles, $P(s)$, and a CO-gas flux from the nucleus surface, $Q$, given by (Fulle et al. 2020)

$$
\begin{aligned}
& P(s)=P_{0} f(s) \exp \left[-\frac{T_{0}}{T_{\mathrm{s}}-s \nabla T}\right] \\
& Q=\frac{14 r P(R)}{3 R} \sqrt{\frac{2 m}{\pi k\left(T_{\mathrm{s}}-R \nabla T\right)},}
\end{aligned}
$$

where $s$ is the depth from the nucleus surface and $f(s)=$ $1-\left(1-\frac{s}{R}\right)^{4}$ for $s \leq R, f(s)=1$ elsewhere. In the range of temperatures $40 \leq T_{\mathrm{s}} \leq 50 \mathrm{~K}$ of the nucleus surface, the values $P_{0}=1.73 \times 10^{10} \mathrm{~Pa}$ and $T_{0}=942 \mathrm{~K}$ fit the available CO-ice sublimation data best (Fray \& Schmitt 2009). $\nabla T$ is the nucleus average gradient of the temperature at $P \geq 0.1 \mathrm{~Pa}$, $r \approx 50 \mathrm{~nm}$ and $R \approx 5 \mathrm{~mm}$ are the radii of the grains of which cometary dust consists (Levasseur-Regourd et al. 2018; Mannel et al. 2019; Güttler et al. 2019) and of the pebbles of which cometary nuclei consist (Blum et al. 2017; Fulle et al. 2020), $m$ is the mass of the CO molecule, and $k$ is the Boltzmann constant (all symbols are listed in Table 1).

The value of $T_{\mathrm{s}}$ is given by the energy balance equation,

$(1-A) I_{\odot} \cos \theta r_{\mathrm{h}}^{-2}=\epsilon \sigma T_{\mathrm{s}}^{4}+\lambda_{\mathrm{s}} \nabla T+\Lambda Q$,

where $A$ is the nucleus Bond albedo (e.g., $A=1.2 \%$ measured at 67P; Fornasier et al. 2015), $I_{\odot}$ is the solar flux at the heliocentric distance of Earth, $\theta$ is the solar zenithal angle, $r_{\mathrm{h}}$ is the heliocentric distance of the nucleus in astronomical units, $\epsilon \approx 0.9$ is the emissivity, $\sigma$ is the Stefan-Boltzmann constant, $\lambda_{\mathrm{s}}$ is the heat conductivity inside the nucleus, and $\Lambda=2.27 \times 10^{5} \mathrm{~J} \mathrm{~kg}^{-1}$ is the latent heat of sublimation of CO ice (Huebner et al. 2006).

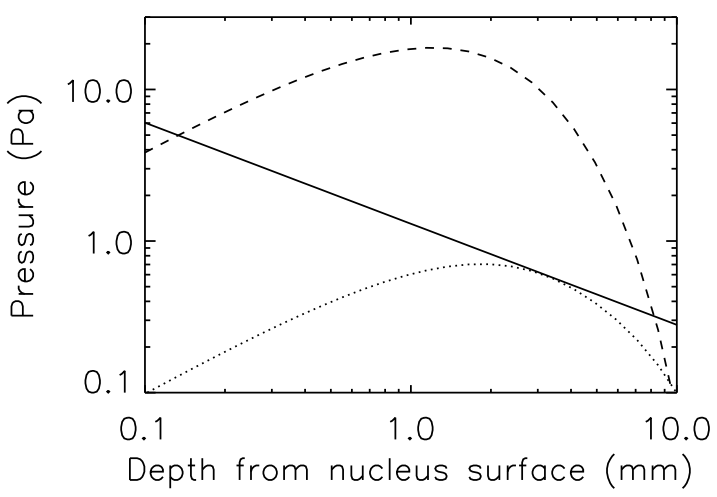

Fig. 1. Pressure $P$ inside the pebbles vs. depth $s$. The continuous line shows the tensile strength, $S$, bonding a homogeneous aggregate of dust grains. The dashed line represents $P$ at $T_{\mathrm{s}}=48 \mathrm{~K}$ and $\nabla T=12 \mathrm{~K} \mathrm{~cm}^{-1}$, which crosses $S$ at $s_{\mathrm{m}}=0.12 \mathrm{~mm}$ and $s_{\mathrm{M}}=8 \mathrm{~mm}$. The dotted line shows $P$ at $T_{\mathrm{s}}=40 \mathrm{~K}$ and $\nabla T=4 \mathrm{~K} \mathrm{~cm}^{-1}$ and is tangent to $S$ at $s_{\mathrm{m}}=$ $s_{\mathrm{M}}=3 \mathrm{~mm}$. For every value of $T_{\mathrm{s}}$, the nucleus at the depth $s_{\mathrm{M}}$ maintains a temperature $T_{\mathrm{s}}-\nabla T s_{\mathrm{M}}=38.5 \mathrm{~K}$.

The values of $\lambda_{\mathrm{s}}$ and $\nabla T$ are provided by (Fulle et al. 2020)

$\nabla T=\frac{\sqrt{\Lambda Q / \sigma}}{8\left(T_{\mathrm{s}}-R \nabla T\right) R}$

$\lambda_{\mathrm{s}}=\frac{32}{3} \sigma R\left(T_{\mathrm{s}}-R \nabla T\right)^{3}$

because in a pebble-made nucleus the heat conductivity is dominated by the radiation among the pebbles (Blum et al. 2017). Equations (1)-(5) must be solved altogether, fixing $T_{\mathrm{s}}, \nabla T, \lambda_{\mathrm{s}}$, and $Q$. The tensile strength, $S$, which bonds the superficial dust particles to the pebble, is $S=13 s^{-2 / 3} \mathrm{mPa}$ (with $s$ in meters; Skorov \& Blum 2012, Fig. 1). Because the ejected dust is detached from the nucleus surface, we can identify the dust size with the depth $s . P \geq S$ defines the minimum, $s_{\mathrm{m}}$, and maximum, $s_{\mathrm{M}}$, ejected dust sizes. The nucleus at the depth $s_{\mathrm{M}}$ maintains a constant temperature $T_{\mathrm{s}}-s_{\mathrm{M}} \nabla T \approx 38.5 \mathrm{~K}$ (Fig. 1), so that dust ejection occurs in thermal quasi-equilibrium. Therefore the rates at which the nucleus is eroded, $E$, and is CO-depleted, $D$, are (Fulle et al. 2020)

$\begin{aligned} E & =\frac{32(1+\delta) \sigma R\left(T_{\mathrm{s}}-s_{\mathrm{M}} \nabla T\right)^{3}}{3\left(\frac{1}{2}+\delta\right) \rho_{\mathrm{d}} c_{\mathrm{p}} s_{\mathrm{M}}} \\ D & =\frac{(1+\delta) \gamma Q}{\rho_{\mathrm{n}}}\end{aligned}$

where $\rho_{\mathrm{d}} \approx 800 \mathrm{~kg} \mathrm{~m}^{-3}$ is the average bulk density of the refractory particles (Fulle et al. 2017), $c_{\mathrm{p}} \approx 10^{3} \mathrm{~J} \mathrm{~kg}^{-1} \mathrm{~K}^{-1}$ is the heat capacity of the pebbles (Blum et al. 2017), $\delta$ and $\gamma$ are the average refractory-to-all-ices and all-ices-to- $\mathrm{CO}$ mass ratios in the nucleus, respectively, and $\rho_{\mathrm{n}}=538 \mathrm{~kg} \mathrm{~m}^{-3}$ is the average bulk density of the nucleus (Pätzold et al. 2019). Here, with all-ices we mean $\mathrm{H}_{2} \mathrm{O}, \mathrm{CO}_{2}$, and $\mathrm{CO}$ ices, where the first two behave as refractories at $T<50 \mathrm{~K}$ and have a bulk density close to half of that of the compacted refractories (Fulle et al. 2017). Measurements in comets Hale-Bopp and Hyakutake provided the gas mass fractions $\mathrm{CO} / \mathrm{H}_{2} \mathrm{O} \approx 40 \%$ and $\mathrm{CO}_{2} / \mathrm{H}_{2} \mathrm{O} \approx 15 \%$ (Bockelee-Morvan et al. 2004), suggesting $\gamma \approx 4$.

When $D<E$, the CO-driven activity never stops because the $\mathrm{CO}$-gas diffusion erodes the superficial pebbles before they become $\mathrm{CO}$-depleted, exposing the $\mathrm{CO}$-ice-rich ones underneath. In contrast, when $D>E$, the CO-driven activity stops because the $\mathrm{CO}$-depletion builds up an insulating crust before it is eroded by the dust ejection. 


\section{Discussion}

The activity onset occurs when the $P$ curve in Fig. 1 is tangent to the $S$ line, that is when Eqs. (1) $-(5)$ provide $T_{\mathrm{s}}=40 \mathrm{~K}$, $\nabla T=4 \mathrm{~K} \mathrm{~cm}^{-1}, Q=10^{-7} \mathrm{~kg} \mathrm{~m}^{-2} \mathrm{~s}^{-1}, \lambda_{\mathrm{s}}=1.710^{-4} \mathrm{~W} \mathrm{~m}^{-1} \mathrm{~K}^{-1}$, and $s_{\mathrm{m}}=s_{\mathrm{M}}=3 \mathrm{~mm}$. These values in Eq. (3) provide $r_{\mathrm{h}} \approx 85 \mathrm{au}$ if $\cos \theta \approx 1$. The observed activity onset at $r_{\mathrm{h}} \approx 26$ au suggests $\cos \theta \approx 0.1$, that is, a nucleus that is inactive in all areas with $0.1 \ll \cos \theta \leq 1$. For instance, if the nucleus spin axis points to the Sun, then the active area would be a tight equatorial belt. Conversely, if the nucleus spin axis has a low obliquity, then the active area would be a small polar spot. The orbital period of $\approx 3$ Myr makes the orbit sensitive to galactic tides and perturbations by Jupiter, with a nucleus spin obliquity probably different in the past, when subsolar activity may have covered all the areas that now have $0.1 \ll \cos \theta \leq 1$ of inactive fallout. A fallout of all the dust ejected beyond 26 au from the Sun is improbable because the $\mathrm{CO}$ flux increases by a factor 10 only from the activity onset up to the observations. According to Eq. (6), the erosion rate remains about constant (within a factor 3 ) since the activity onset, explaining the steady brightness slope of the $\mathrm{C} / 2017 \mathrm{~K} 2$ dust coma (Jewitt et al. 2019).

With $\cos \theta \approx 0.1$, Eqs. (1)-(5) at $r_{\mathrm{h}}=14$ au provide $T_{\mathrm{s}}=48 \mathrm{~K}, \nabla T=12 \mathrm{~K} \mathrm{~cm}^{-1}, Q=10^{-6} \mathrm{~kg} \mathrm{~m}^{-2} \mathrm{~s}^{-1}, \lambda_{\mathrm{s}}=$ $2.3 \times 10^{-4} \mathrm{~W} \mathrm{~m}^{-1} \mathrm{~K}^{-1}, s_{\mathrm{m}}=0.12 \mathrm{~mm}$, and $s_{\mathrm{M}}=8 \mathrm{~mm}$ (Fig. 1). The obtained value of $s_{\mathrm{m}}$ is consistent with the available observations at $r_{\mathrm{h}}=14$ au (Jewitt et al. 2019). The CO-driven activity never stops if $\delta \leq 4.5$ in Eqs. (6) and (7) (i.e., if the refractory-to-water-ice mass ratio is $\leq 7$ ), in which case $D \leq E=$ $3 \mathrm{~mm} \mathrm{day}^{-1}$. When no dust fallout is assumed, the observed dustloss rate $Q_{\mathrm{d}} \approx 200 \mathrm{~kg} \mathrm{~s}^{-1}$ (Jewitt et al. 2019) implies a nucleus active area of $(1+\delta) Q_{\mathrm{d}} /\left[\left(\frac{1}{2}+\delta\right) \rho_{\mathrm{d}} E\right] \approx 7 \mathrm{~km}^{2}$, with a COgas loss rate of $\approx 7 \mathrm{~kg} \mathrm{~s}^{-1}$. However, the computed $Q$-values are too low to drag all the dust of sizes $s_{\mathrm{m}} \leq s \leq s_{\mathrm{M}}$ up to the escape velocity (Zakharov et al. 2018). If the fallout amounts to $\geq 30 \%$ of the eroded dust mass (a fraction much lower than occurred on 67P/Churyumov-Gerasimenko; Fulle et al. 2019), then the nucleus active area becomes $\geq 10 \mathrm{~km}^{2}$, with a CO-gas loss rate $\geq 10 \mathrm{~kg} \mathrm{~s}^{-1}$, and a dust-to-gas mass ratio in the lost material $\leq 20$, consistent with the average dust-to-CO-ice mass ratio $(1+\delta) \gamma-1 \leq 21$ in the nucleus (Fulle et al. 2019).

The CO-driven erosion of $3 \mathrm{~mm} \mathrm{day}^{-1}$ implies that cometary planetesimals, with radius $\leq 50 \mathrm{~km}$, a pristine season-independent activity, and buildup at heliocentric distances $<85$ au by the gravitational collapse of centimeter-sized pebbles (Blum et al. 2017), were completely eroded within $50 \mathrm{kyr}$ after the protoplanetary disk became optically thin to solar radiation. This erosion lifetime is $10^{3}$ times shorter than the timescale of catastrophic collisions that occurred in the first $0.5 \mathrm{Gyr}$ after planet formation (Morbidelli \& Rickman 2015), which in turn excludes that comets were destroyed by collisions before a complete erosion. This suggests a natural source of the observed $\mathrm{CO}$ and debris disks (Hughes et al. 2017), that is, CO-driven cometary activity, which eroded most cometary planetesimals into millimeter-sized dust, with a Poynting-Robertson timescale of the orbital collapse of $\approx 1$ Gyr (Burns et al. 1979). Because cometary planetesimals probably formed after the giant planets (Fulle \& Blum 2017), the only ones surviving into comets were those that closely encountered migrating planets before a complete erosion, with a storage into orbits of perihelion $>85 \mathrm{au}$, where the timescale of catastrophic collisions is $>5$ Gyr (Morbidelli \& Rickman 2015). It follows that comets observed today cannot be products of catastrophic collisions, consistent with the constraints provided by the Rosetta mission (Fulle \& Blum 2017).

\section{Conclusions}

The activity model based on gas diffusion inside the pebbles of which cometary nuclei consist (Fulle et al. 2020) was applied here to comet $\mathrm{C} / 2017 \mathrm{~K} 2$ PANSTARRS, whose activity is driven by $\mathrm{CO}$-ice sublimation assuming $\mathrm{CO}$ as pure ice and neglecting the fraction that is trapped in other ices and clathrates. We list our results below.

(i) CO-gas pressure inside the pebbles reaches $20 \mathrm{~Pa}$ and erodes the nucleus surface into ejected dust (composed of refractories, $\mathrm{H}_{2} \mathrm{O}$ ice, and $\mathrm{CO}_{2}$ ice), without invoking other processes to explain the observed dust coma (Jewitt et al. 2019).

(ii) CO-driven activity onset occurs up to heliocentric distances of $85 \mathrm{au}$, ejecting dust of $\approx 3 \mathrm{~mm}$ size.

(iii) The activity onset of $\mathrm{C} / 2017 \mathrm{~K} 2$, observed at $\approx 26 \mathrm{au}$ (Jewitt et al. 2019), suggests a low obliquity of the nucleus spin axis if the activity mainly occurs in a polar summer.

(iv) At $14 \mathrm{au}$, the smallest size of the ejected dust is $\approx 0.1 \mathrm{~mm}$, consistent with observations (Jewitt et al. 2019).

(v) The observed dust-loss rate of $\approx 200 \mathrm{~kg} \mathrm{~s}^{-1}$ (Jewitt et al. 2019) implies a fallout $\geq 30 \%$, a nucleus surface active area $\geq 10 \mathrm{~km}^{2}$, and a CO-gas loss rate $\geq 10 \mathrm{~kg} \mathrm{~s}^{-1}$.

(vi) CO-driven activity never stops if the average refractoryto-all-ices mass ratio in the nucleus is $\delta \leq 4.5$ for a nucleus allices-to-CO mass ratio $\gamma \approx 4$, as observed in comets Hale-Bopp and Hyakutake. This $\delta$-value is consistent with the value found for comet 67P (Fulle et al. 2020) and with the constraints given by pebble accretion models in protoplanetary disks (Lorek et al. 2016), namely $\delta \geq 3$.

(vii) The CO-driven erosion lifetime of cometary planetesimals with $\delta \leq 4.5$ and a pristine season-independent activity is a factor $10^{3}$ shorter than the timescale of catastrophic collisions (Morbidelli \& Rickman 2015). This means that comets observed today cannot be products of catastrophic collisions (Fulle \& Blum 2017).

Acknowledgements. We thank an anonymous referee for having improved the first version of this letter. Part of this research was supported by the ESA Express Procurement (EXPRO) RFP for IPL-PSS/JD/190.2016 and by the Italian Space Agency (ASI) within the ASI-INAF agreements I/032/05/0 and I/024/12/0. J.B. thanks the Deutsche Forschungsgemeinschaft for support under grant BL298/261 as part of the international CoPhyLab collaboration. CoPhyLab is jointly funded through DFG (Germany), FWF (Austria) and SNF (Switzerland).

\section{References}

Blum, J., Gundlach, B., Krause, M., et al. 2017, MNRAS, 469, S755 Burns, J. A., Lamy, P. L., \& Soter, S. 1979, Icarus, 40, 1

Bockelee-Morvan, D., Crovisier, J., Mumma, M. J., \& Weaver, H. A. 2004, in Comets II, eds. M. C. Festou, H. U. Keller, \& H. A. Weaver (Tucson: Univ. of Arizona Press, Tucson), 391

Fornasier, S., Hasselmann, P. H., Barucci, M. A., et al. 2015, A\&A, 583, A30

Fray, N., \& Schmitt, B. 2009, Plan. Space Sci., 57, 2053

Fulle, M., \& Blum, J. 2017, MNRAS, 469, S39

Fulle, M., Della Corte, V., Rotundi, A., et al. 2017, MNRAS, 469, S45

Fulle, M., Blum, J., Green, S., et al. 2019, MNRAS, 482, 3326

Fulle, M., Blum, J., Rotundi, A., et al. 2020, MNRAS, 493, 4039

Güttler, C., Mannel, T., Rotundi, A., et al. 2019, A\&A, 630, A24

Huebner, W. F., Benkhoff, J., Capria, M. T., et al. 2006, The International Space Science Institute, Bern, Switzerland (Noordwijk, The Netherlands: ESA Publications Division)

Hughes, A. M., Lieman-Sifry, J., Flaherty, K. M., et al. 2017, ApJ, 839, 86 Jewitt, D., Agarwal, J., Hui, M.-T., et al. 2019, AJ, 157, 65

Levasseur-Regourd, A.-C., Agarwal, J., Cottin, H., et al. 2018, Space Sci. Rev., 214,64

Lorek, S., Gundlach, B., Lacerda, P., \& Blum, J. 2016, A\&A, 587, A128

Mannel, T., Bentley, M. S., Boakes, P. D., et al. 2019, A\&A, 630, A26

Morbidelli, A., \& Rickman, H. 2015, A\&A, 583, A43

Pätzold, M., Andert, T. P., Hahn, M., et al. 2019, MNRAS, 483, 2337

Snodgrass, C., \& Jones, G. H. 2019, Nat. Comm., 10, 5418

Skorov, Y. V., \& Blum, J. 2012, Icarus, 221, 1

Zakharov, V. V., Ivanovski, S. L., Crifo, J. F., et al. 2018, Icarus, 312, 121 